\title{
Routine examination of the newborn and maternal satisfaction: a randomised controlled trial
}

\author{
D Wolke, S Dave, J Hayes, J Townsend, M Tomlin
}

\begin{abstract}
Objective: To determine whether the routine examination of the newborn by a midwife compared with a junior paediatrician (SHO) affects maternal satisfaction with this examination.

Methods: Randomised controlled trial: 826 mother and baby pairs in a district general hospital in south east England were randomised to a paediatric $\mathrm{SHO}$ or a midwife for the routine newborn examination. Maternal satisfaction with the examination was analysed in relation to intervention group, process, and background variables.

Results: Some $81 \%$ of mothers reported that they were satisfied or very satisfied with the newborn examination. Mothers assigned to a midwife were more satisfied with the newborn examination (crude odds ratio (OR) 0.54 (95\% confidence interval (Cl) 0.39 to 0.75$), p<0.001$ ). However, after provision of health education during the examination, continuity of care provided, and history of miscarriage had been controlled for, status of examiner was no longer related to maternal satisfaction (adjusted OR $0.82(95 \% \mathrm{Cl} 0.57-1.20), \mathrm{NS})$. The discussion of healthcare issues by the examiner ladjusted OR $0.49(95 \% \mathrm{Cl} 0.34$ to 0.70$), \mathrm{p}<0.001)$ and continuity of care (adjusted OR $0.43(95 \%$ $\mathrm{Cl} 0.23$ to 0.81$), \mathrm{p}<0.01$ ) were both related to enhanced satisfaction, and history of miscarriage (adjusted OR 1.61 (1.08 to 2.40), p < 0.05) was associated with lower maternal satisfaction with the newborn examination. Midwives (61\%) were more likely than $\mathrm{SHOs}(33 \%)$ to discuss healthcare issues, such as feeding, sleeping, and skin care.

Conclusions: Mothers were more likely to be satisfied with the newborn examination by a midwife than an $\mathrm{SHO}$ because midwives were more likely to discuss healthcare issues during the examination and were able to provide continuity of care. However, midwife examinations according to exclusion criteria agreed with trial midwives excluded half of all newborns, and criteria may have to be reconsidered for practice implementation.
\end{abstract}

See end of article for
authors' affiliations

Correspondence to: Professor Wolke,

Department of Psychology,

University of Hertfordshire,

GB-Hatfield, Herts

AL10 9AB, UK:

D.F.H.Wolke@herts.ac.uk

Accepted

18 December 2001
A detailed examination of the newborn early in the neonatal period is recommended as a core component of Child Health Surveillance. ${ }^{1}$ Although some doubt has been raised about the necessity of the newborn examination, ${ }^{23}$ it is widely accepted as good practice and expected by parents. ${ }^{145}$ With the exception of screening for developmental dysplasia of the hips, there is no national UK policy on the examination. ${ }^{6}$ Recommended components of the neonatal examination include history taking, physical examination, health education, and parental assurance. ${ }^{137}$ Traditionally this has been carried out by junior paediatricians in training usually at SHO level. The value of the examination as a screening tool has been questioned by several authors, as less than $30 \%$ of congenital heart defects or hip abnormalities are detected during the examination. ${ }^{8}{ }^{9}$ Neither maternal satisfaction with the examination nor the provision of health education and parental reassurance have yet been evaluated.

Owing to recent changes in the organisation of maternity care and training, and the reduction in the number of hours worked by junior doctors, extension of the role of the midwife has been advocated..$^{10-12}$ These developments have led to the introduction of the post-registration course in the examination of the newborn approved by the English National Board, ${ }^{13}$ which is open to midwives, health visitors, and doctors. Although Hall ${ }^{13}$ concluded that a midwife could undertake the examination provided that clear guidelines, adequate training, and paediatric support are provided, there have been no systematic evaluations of the quality of the examination performed by midwives compared with junior doctors.

The focus of current health service policy is the need to develop a consumer orientation and to provide services that are patient centred. ${ }^{10}{ }^{14} 15$ An important criterion of the quality of healthcare services is patient or customer satisfaction. ${ }^{16-20}$ Satisfaction is a reflection of the degree of congruency between a customer's expectations of ideal care and his/her perception of the real care received. ${ }^{1821}$ Components that influence patient satisfaction are perceived technicalprofessional competence of the healthcare provider, relationship to and communication with the patient, and provision of information to the patient. ${ }^{21-26}$ Hall $^{3}$ proposed that, for the evaluation of different professional groups that carry out the newborn examination, parental satisfaction and provision of information should be considered as a major outcome.

As part of a wider evaluation of the safety and cost effectiveness of the newborn examination, a randomised controlled trial was conducted to determine whether examination by the midwife or senior house officer ( $\mathrm{SHO}$ ) affects maternal satisfaction (clinical objective). Secondary analysis was undertaken to evaluate whether any specific factors during the newborn examination (health education, problems identified, duration of examination) can explain any group differences in maternal satisfaction ratings. ${ }^{27}$

\section{METHODS}

\section{Study population}

The study was carried out in south east England in a district general hospital with about 3000 deliveries a year. Recruitment took place between May 1999 and August 2000. Mothers in the postnatal ward between the hours of 0800 and 1600 on at least one of the minimum five days of recruitment a week were potential study participants. 


\section{Intervention}

The randomised interventions were routine examination of the newborn by a paediatric SHO or a community midwife. All community midwives conducting the examination had completed the English National Board (ENB) N96 postregistration course in examination of the newborn. SHO training for the newborn examination was more informal and had been carried out by registrars or a consultant paediatrician.

Babies included in the study were those that would have normally been eligible for a midwife examination, according to criteria formulated by senior paediatric and midwifery staff at the hospital. Exclusion criteria were: (a) maternal problems, including chronic disease, infection, drug dependency, drugs with known side effects taken during pregnancy, family history of genetic or inherited diseases; $(b)$ infant problems, including emergency or elective caesarean section under general anaesthetic, instrumental or operative delivery with perinatal complications, Apgar score $<5$ at one minute and/or resuscitation required at birth, gestation under 37 weeks, birth weight below $2500 \mathrm{~g}$ or above $4500 \mathrm{~g}$, abnormalities detected antenatally or at birth needing follow up, jaundice, problems since birth requiring medical investigation, abnormal neurological responses, dysmorphic features, admissions to the special care baby unit, and history of symptomatic meconium stained liquor. All other births were potential candidates for inclusion in the study. We estimated that about a half of all births would be eligible for the trial. Eligibility for the trial was assessed by the midwife managing the postnatal ward as part of the usual procedure for admitting the healthy newborn.

Newborn examinations were usually carried out 6-24 hours after birth, which is considered an optimal period by paediatric and midwifery management at the hospital, and is also the time recommended by Hall. ${ }^{1}$ Examinations by midwives were carried out either in hospital (84\%) or at the mother's home ( $16 \%$ ) depending on the duration of her postnatal stay in hospital. All examinations by SHOs were carried out in hospital. Referrals as a result of the examination were recorded by the examiner on the specially designed newborn examination form. Referrals to a registrar, consultant, or outpatient department were followed subsequently through the medical notes for outcome. The study protocol was reviewed by the NHS Trust ethics committee.

\section{Randomisation}

A research midwife working on the postnatal ward provided written information and explained the study to eligible women. Women who agreed to participate were asked to provide signed consent. Each consenting mother and baby pair were individually assigned to an intervention group using random numbers generated by a coordinator of the study using a statistical software package. An identification sticker was placed on the front cover of the infant's notes to ensure that babies were examined by the allocated examiner. Examiners were therefore not blinded to trial participation, and it was not possible to conceal the identity of the examiner from the mothers.

\section{Outcome measure}

The primary outcome measure was maternal satisfaction with the newborn examination. As no tool existed to measure maternal satisfaction with the routine examination of the newborn, a self completion questionnaire was developed by the multidisciplinary research team (comprising psychologists, a health economist, paediatric and midwifery staff, health services researchers, and a statistician), which was piloted on 60 mothers. As well as measuring overall satisfaction with the examination, the questionnaire was designed to determine the degree of satisfaction with specific
For each of the statements below please show by circling one number how satisfied or dissatisfied you feel about the way your baby was examined (the negative numbers -3 to -1 indicate levels of dissatisfaction, and the positive numbers +1 to +3 indicate levels of satisfaction).

How do you feel about:

1. how the examiner introduced him/herself?

2. how the examiner explained the reason for the examination?

3. how the examiner handled your baby?

4. how thoroughly your baby was examined?

5. how friendly the examiner was?

6. opportunities you had to ask questions?

7. Overall how satisfied were you with the examination?

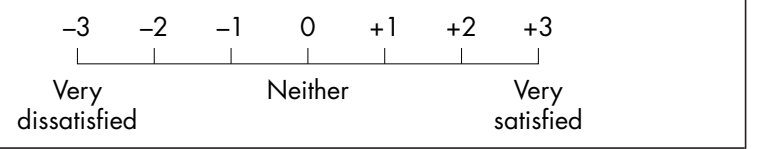

Figure 1 Items on the questionnaire identified as relating to maternal satisfaction.

aspects, including the manner and communication style of the examiner, information given, and handling of the baby, using a seven point Likert-type scale ranging from "very dissatisfied" (-3) to "very satisfied" (+3) (fig 1). Satisfaction with care during labour and since delivery, maternal mood, and bonding with the baby were also assessed.

The mother was handed the questionnaire and a prepaid envelope after the newborn examination had been carried out. Participants had the option of either leaving their completed questionnaire in a specially placed box on the ward reception or completing it at home. Non-respondents were sent a written reminder, followed by a telephone call. Respondents and non-respondents ${ }^{28}$ were compared with respect to sociodemographic, obstetric, and medical data, which were collected from mother and infant hospital records.

Principal components analysis with varimax rotation on all respondents with a complete data set $(n=645)$ was used to indicate which questions assessed satisfaction with aspects of the newborn examination. The analysis identified seven of the questions, loading on a first factor, that assessed satisfaction with the newborn examination. These items in the scale loaded relatively equally (loading: 0.82-0.90), so mean scores were calculated by summing the actual scores for the respective questions and dividing by seven. A Cronbach's $\alpha$ coefficient of 0.94 indicated that the mean satisfaction scale had high internal reliability. Two other factors were identified; these represented the mood of the mother and bonding with the baby (factor 2), and satisfaction with care during and since labour and delivery (factor 3). Factors 2 and 3 had only weak correlations with the newborn satisfaction scale (Spearman's rank correlation $(r)=0.15, \mathrm{p}<0.001$; and $r=-0.09$, $\mathrm{p}=0.02$ for factors 2 and 3 respectively), indicating that the satisfaction scale had high discriminatory validity.

\section{Eligibility}

A retrospective census was conducted on 100 consecutive births at the study hospital, to determine the potential number of babies eligible for midwife examination according to the exclusion criteria. 


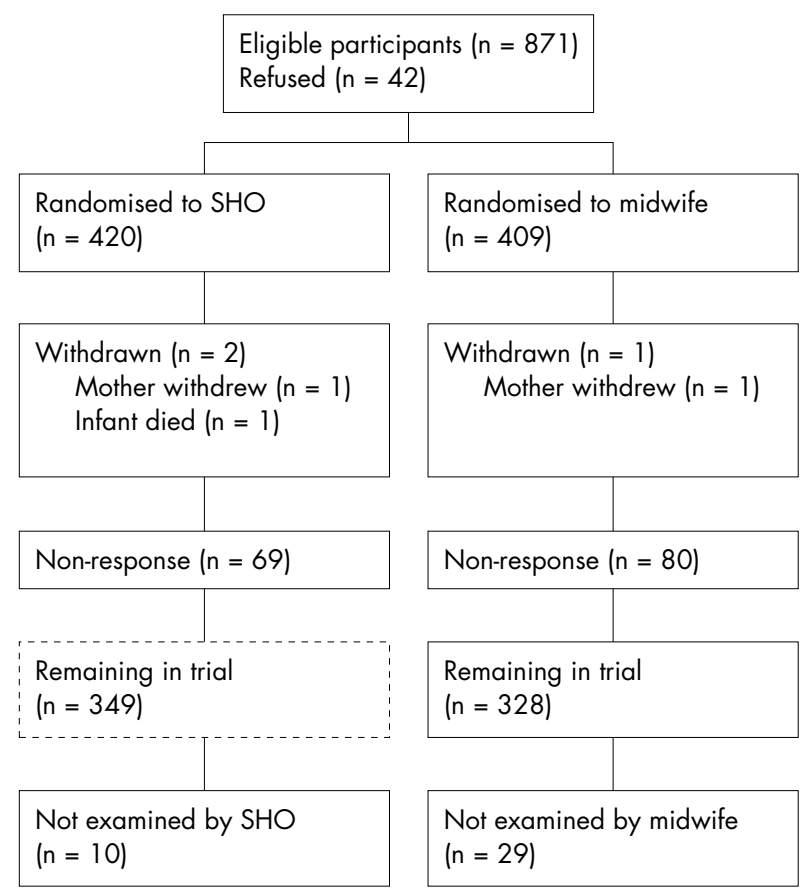

Figure 2 Participant flow through trial.

\section{Statistical analysis}

Analysis of a pilot study indicated that around 1000 subjects could be recruited within 15 months at the study site. We estimated that this sample size would be adequate to detect about a $9 \%$ difference in satisfaction levels between the two study groups at $80 \%$ power and $5 \%$ significance.

Results from the pilot study indicated that satisfaction scores were skewed, with a high proportion of women reporting that they were satisfied or very satisfied. A decision was made a priori to dichotomise the distribution into two categories: low satisfaction scores (33\% lowest scoring of sample) and medium to high satisfaction scores. Analysis was by intention to treat using a maximum likelihood logistic regression model to predict low satisfaction. Sociodemographic, obstetric, and health variables (table 1) were tested as potential confounders, and examination specific variables (table 3: healthcare issues discussed, problems detected, length of examination, newborn examined by midwife who clerked first antenatal appointment, and place of examination) were tested as potential factors explaining intervention group differences in satisfaction. Modelling was aided using likelihood ratio tests. The final model included predictors significantly and independently associated with maternal satisfaction. This analysis was repeated on an actual treated by SHOs $v$ midwives basis (explanatory analysis). Statistical analysis was performed using Stata 6.0 software, and two sided $\alpha$ values are reported.

\section{RESULTS}

Figure 2 shows participant flow through the study. Forty two out of 871 (5\%) women approached refused to participate. Of those that consented, 420 were randomised to examination by an SHO and 409 were randomised to examination by a midwife. Three women $(0.4 \%)$ were withdrawn from the study, two because they so requested, and the other because her baby had died. Mother and baby hospital documentation was obtained for $98 \%$ of the study participants; however, a number of records had data missing. Those that consented to participate in the study had a mean (SD) age of 30 (5) years $(\mathrm{n}=823) ; 87 \%$ were white $(\mathrm{n}=799), 42 \%$ were primigravida $(\mathrm{n}=822), 74 \%$ were house owners $(\mathrm{n}=749)$, and $77 \%$ had delivered by spontaneous vaginal delivery with no instrumental intervention $(\mathrm{n}=825)$.

A total of $677(82 \%)$ women completed and returned the questionnaire. Ten of the 349 (3\%) babies assigned to an SHO were in fact examined by a midwife, and 29 of the 328 (9\%) subjects assigned to a midwife were examined by an SHO. In most cases, this was because the allocated examiner was not available at the time of discharge from hospital. Table 1 shows the sociodemographic, obstetric, and health profile of respondents and early feeding choice distributions, by intention to treat by SHO or midwife. A comparison of these variables between respondents $(n=677)$ and non-respondents $(\mathrm{n}=149)$ was carried out. Of 16 variables tested (table 1$)$, only housing tenure and ethnic status were related to questionnaire response. Respondents were significantly more likely to be house owners (471/619 (76\%) v 83/131 (63\%), $\mathrm{p}=0.002)$, and were more likely to be white $(582 / 653(89 \%) v$

Table 1 Sociodemographic, obstetric, medical, and early infant feeding profile of respondents, by intention to treat

\begin{tabular}{|c|c|c|c|}
\hline \multirow[b]{2}{*}{ Variables } & \multicolumn{2}{|c|}{ Intention to treat } & \multirow[b]{2}{*}{ Total } \\
\hline & $\mathrm{SHO}$ & Midwife & \\
\hline Maternal age: above 30 years & $180 / 347(52)$ & $176 / 330(53)$ & $356 / 677(53)$ \\
\hline Marital status: married & $254 / 324(78)$ & $233 / 317(74)$ & $487 / 641(76)$ \\
\hline Ethnic status: non-white & $32 / 337(10)$ & $39 / 316(12)$ & $71 / 653(11)$ \\
\hline Housing tenure: owner & $241 / 320(75)$ & $230 / 299(77)$ & $471 / 619(76)$ \\
\hline Parity: primiparous & $158 / 345(46)$ & $121 / 329(37)$ * & $279 / 674(41)$ \\
\hline History of miscarriage: yes & $80 / 346(23)$ & $62 / 329(19)$ & $142 / 675(21)$ \\
\hline Smoking status at first antenatal visit: yes & $49 / 343(14)$ & $50 / 326(15)$ & $99 / 669(15)$ \\
\hline Alcohol consumption status at first antenatal visit: yes & $70 / 341(21)$ & $83 / 325(26)$ & $153 / 666(23)$ \\
\hline Mode of delivery: spontaneous vaginal delivery & $247 / 347(71)$ & $272 / 330(82) * * *$ & $519 / 677$ (77) \\
\hline Median length of labour: minutes & $346(n=316)$ & $349(n=314)$ & $346(n=630)$ \\
\hline$(95 \% \mathrm{Cl})$ & (312 to 396$)$ & (309 to 385 ) & (317 to 378$)$ \\
\hline Epidural or pethidine administered: yes & $138 / 346(40)$ & $123 / 330(37)$ & $261 / 376$ (39) \\
\hline Complications during labour: yes & $181 / 323(56)$ & $156 / 302(52)$ & $337 / 625(54)$ \\
\hline Past medical history of problems: yes & $235 / 345(68)$ & $218 / 324(67)$ & $453 / 669(68)$ \\
\hline Past history of depression: yes & $34 / 345(10)$ & $33 / 324(10)$ & $67 / 669(10)$ \\
\hline thas put baby to breast: yes & $273 / 345(79)$ & $265 / 321(83)$ & $538 / 666(81)$ \\
\hline †Feeding intentions: solely breastfeeding & $158 / 343(46)$ & $149 / 327(46)$ & $307 / 670(46)$ \\
\hline
\end{tabular}

Unless indicated otherwise, values in parentheses are percentages ${ }^{*} \mathrm{p} \leqslant 0.05,{ }^{* *} \mathrm{p} \leqslant 0.001$ compared with those examined by SHO. †Reported by the mother on the maternal satisfaction questionnaire. $\mathrm{SHO}$, Senior house officer. 
Table 2 Relative distribution of low and medium/high satisfaction with the newborn examination, by intention to treat $(n=645)$

\begin{tabular}{llll}
\hline & \multicolumn{2}{l}{ Intention to treat (\%) } & \\
\cline { 2 - 3 } Relative satisfaction score & SHO & Midwife & Total \\
\hline Low & $133(40)$ & $83(27)$ & $216(33)$ \\
Medium/high & $199(60)$ & $230(73)$ & $429(67)$ \\
Total & $332(100)$ & $313(100)$ & $645(100)$ \\
\hline
\end{tabular}

Crude OR (low satisfaction) $=0.54(95 \% \mathrm{Cl} 0.39$ to 0.75 , $p \leqslant 0.001)$.

$\mathrm{SHO}$, Senior house officer.

$113 / 146(77 \%), p<0.001)$ than non-respondents. Respondents and non-respondents did not significantly differ with respect to other sociodemographic, obstetric, and medical factors.

Overall satisfaction with the newborn examination was high; $81 \%$ of women $(85 \%$ in the midwife group and $78 \%$ in the SHO group) reported a mean score of +2 or +3 (high or very high satisfaction).

\section{Differences by intention to treat}

Table 2 shows the relative distribution of low and medium/ high satisfaction by intention to treat. Although overall satisfaction was high, women in the midwife group were significantly less likely to report a low satisfaction score than were women in the SHO group (midwives 27\% v SHOs $40 \%: \chi^{2}$ $($ df 1$)=11.3$; OR $0.54,95 \%$ CI 0.39 to $0.75, \mathrm{n}=645)$.

\section{Confounders and examination specific variables}

Table 1 shows that despite randomisation, the SHO group contained more women who were primiparous $\left(\chi^{2}=5.3\right.$, $\mathrm{p}=0.02)$ and fewer women with spontaneous vaginal delivery $\left(\chi^{2}=12.4, \mathrm{p}<0.001\right)$.

Four out of five examination specific variables differed between midwives and SHOs (table 3). Almost twice as many midwives as SHOs $(61 \% v 33 \%)$ were reported to have discussed healthcare issues during the newborn examination, with feeding, skin care, jaundice, sleeping, and stools and nappy care being the most commonly discussed issues. The routine newborn examination was longer in the midwife group than in the SHO group (median $15 v 10$ minutes, Wilcoxon rank sum $\mathrm{z}=-3.91, \mathrm{p}<0.001)$. The number of hospital referrals (to a registrar, consultant, or outpatient department) made by midwives and SHOs during the newborn examination for problems requiring further investigation did not differ ( $4 \%$ for both midwives and SHOs). Continuity of care was defined as the same midwife clerking the mother at the initial antenatal visit and conducting the neonatal examination. On an intention to treat basis, 95/318 midwives provided some continuity of care, and none of the SHOs had had contact with the mother or baby before the routine examination. Some babies were examined at home by a midwife rather than in hospital. Of the babies examined by a midwife, 101 were examined at home, 96 of which had been randomised to examination by a midwife and five to SHO examination.

\section{Adjusted model}

Neither parity (crude OR 1.11, 95\% CI 0.80 to $1.55, \mathrm{n}=645$ ) nor type of delivery (crude odds ratio: $1.04,95 \%$ CI 0.70 to $1.53, \mathrm{n}=645$ ) were related to maternal satisfaction with the examination. Of 16 factors (table 1), only history of miscarriage was significantly associated with satisfaction rating, with experience of a miscarriage being associated with lower satisfaction with the newborn examination (crude OR $1.68,95 \%$ CI 1.14 to $2.48, \mathrm{n}=645$; table 4 ).

Women who reported that healthcare issues had been discussed during the examination were less than half as likely to report low satisfaction with the examination (crude OR $0.43,95 \%$ CI 0.30 to $0.60, \mathrm{n}=645)$. Neither length of the examination (crude OR $=0.96,95 \%$ CI 0.92 to $1.00, \mathrm{n}=470$ ) nor examination at home (crude OR $=0.89,95 \%$ CI 0.56 to $1.42, \mathrm{n}=644$ ) were significantly related to maternal ratings of satisfaction. If the midwife who clerked the first antenatal visit also performed the routine newborn examination, women were $67 \%$ less likely to report low satisfaction than if a different midwife or an SHO carried out the examination (crude OR $=0.33,95 \%$ CI 0.18 to $0.58, \mathrm{n}=645$ ).

In table $4 \mathrm{~A}$, the adjusted odds ratios for predicting satisfaction with the newborn examination on an intention to treat basis are shown. After adjustment for history of miscarriage, discussion of healthcare issues, and continuity of care, intention to treat (midwife $v$ SHO) no longer independently predicted satisfaction (adjusted OR $=0.82,95 \%$ CI 0.57 to $1.20, \mathrm{n}=645)$. In contrast, even after adjustment for the other variables, the discussion of healthcare issues during the examination (adjusted OR $=0.49$, 95\% CI 0.34 to 0.70 , $\mathrm{n}=645$ ), a history of miscarriage (adjusted OR $=1.61,95 \%$ CI 1.08 to $2.40, \mathrm{n}=645$ ), and continuity of care (adjusted $\mathrm{OR}=0.43,95 \%$ CI 0.23 to $0.81, \mathrm{n}=645$ ) remained significantly associated with satisfaction with the newborn examination. Whether the baby was examined at hospital or at home was not related to satisfaction.

To ascertain that the discussion of healthcare issues, continuity of care, and experience of previous miscarriage are explanatory factors, the analysis was repeated according to actual treatment received by SHOs $v$ midwives. The results (table 4B) were virtually the same as for the intention to treat analysis.

Table 3 Examination specific variables, by intention to treat

\begin{tabular}{|c|c|c|c|}
\hline \multirow[b]{2}{*}{ Variables } & \multicolumn{2}{|c|}{ Intention to treat } & \multirow[b]{2}{*}{ Total } \\
\hline & $\mathrm{SHO}$ & Midwife & \\
\hline †Healthcare issues discussed during examination: Yes & $111 / 341(33)$ & $199 / 325(61)^{* * *}$ & $310 / 666(47)$ \\
\hline $\begin{array}{l}\S \text { Median length of examination: minutes } \\
(95 \% \mathrm{Cl})\end{array}$ & $\begin{array}{l}10(n=222) \\
(10 \text { to } 10)\end{array}$ & $\begin{array}{l}15(n=269)^{* * *} \\
(11 \text { to } 15)\end{array}$ & $\begin{array}{l}10(n=491) \\
(10 \text { to } 12)\end{array}$ \\
\hline$\S$ Hospital referrals made on examination: Yes & $15 / 349(4)$ & $13 / 326(4)$ & $28 / 675(4)$ \\
\hline Newborn examination by midwife who clerked first antenatal visit: Yes & $\ddagger 5 / 346(2)$ & $95 / 318(30)$ & $100 / 664(15)$ \\
\hline$\dagger$ Newborn examined by midwife at home: Yes & $\mp 5 / 346(3)$ & $96 / 327(29)$ & $101 / 673(16)$ \\
\hline
\end{tabular}

Unless indicated otherwise, values in parentheses are percentages.

$* * * p \leqslant 0.001$ compared with those examined by SHO

†Reported by the mother on the maternal satisfaction questionnaire; łthese five examinations, although allocated to an $\mathrm{SHO}$, were carried out by a midwife; §recorded by the examiner on the newborn examination form. $\mathrm{SHO}$, Senior house officer. 
Table 4 Predicting low maternal satisfaction with the newborn examination using the maximum likelihood logistic regression model

\begin{tabular}{|c|c|c|c|}
\hline Factor & n & Crude odds ratio $(95 \% \mathrm{Cl})$ & Adjusted odds ratio $(95 \% \mathrm{Cl})$ \\
\hline \multicolumn{4}{|l|}{ (A) Intention to treat } \\
\hline Intention to treat (midwife $v \mathrm{SHO}$ ) & $313 / 645$ & $0.54(0.39 \text { to } 0.75)^{* * *}$ & $0.82(0.57$ to 1.20$)$ \\
\hline Healthcare issues discussed (yes) & $297 / 645$ & $0.43(0.30$ to 0.60$) * * *$ & $0.49(0.34$ to 0.70$) * * *$ \\
\hline Previous miscarriage (yes) & $135 / 645$ & $1.68(1.14 \text { to } 2.48)^{* *}$ & $1.61(1.08 \text { to } 2.40)^{*}$ \\
\hline Newborn examination by midwife who clerked first antenatal visit (yes) & $95 / 645$ & $0.33(0.18 \text { to } 0.58)^{* * *}$ & $0.43(0.23$ to 0.81$) * *$ \\
\hline \multicolumn{4}{|l|}{ (B) Actual treatment by $\mathrm{SHOs} v$ midwives } \\
\hline Status of examiner (midwife $v$ SHO) & $291 / 645$ & $0.56(0.40 \text { to } 0.78)^{* * *}$ & $0.91(0.62$ to 1.35$)$ \\
\hline Healthcare issues discussed (yes) & $297 / 645$ & $0.43(0.30$ to 0.60$) * * *$ & $0.48(0.33 \text { to } 0.68)^{* * *}$ \\
\hline Previous miscarriage (yes) & $135 / 645$ & $1.68(1.14 \text { to } 2.48)^{* *}$ & $1.61(1.08 \text { to } 2.40)^{*}$ \\
\hline Newborn examination by midwife who clerked first antenatal visit (yes) & $95 / 645$ & $0.33(0.18 \text { to } 0.58)^{* * *}$ & $0.41(0.22$ to 0.77$)$ * * \\
\hline
\end{tabular}

\section{Eligibility census}

In the eligibility census, the records for $9 \%$ of babies were unobtainable. Of the 91 records that were found, it was determined that 48 (53\%) babies were eligible for midwife assessment according to the criteria formulated by midwives and paediatricians at the trial hospital (see Methods). Fifteen of 48 $(31 \%)$ eligible cases were recruited into the trial.

\section{DISCUSSION}

The satisfaction of mothers was generally high, with some $81 \%$ reporting that they were satisfied or very satisfied with the newborn examination. High satisfaction has been found with other maternal services within the NHS generally. ${ }^{18} 19$ Nevertheless, mothers of newborns examined by midwives were $46 \%$ less likely to have relatively low satisfaction with the newborn examination than those examined by SHOs. However, once continuity of care, history of previous miscarriage, and discussion of healthcare issues during the examination was taken into account, no significant differences in maternal satisfaction with the examination between midwives and SHOs remained. The differences in maternal satisfaction are not explained by the profession of the examiner, but by whether the examiner provided some continuity of care or discussed healthcare issues during the examination.

Midwives but not SHOs may have contact and care for the mother during pregnancy. Walker ${ }^{29}$ proposed that the established relationship between midwife, mother, and child should be built on to create a seamless flow of reassuring care and advice. The newborn examination could be seen as part of that process. In this study, 30\% (95/318) of examinations on an intention to treat basis were performed by a midwife who had met the mother antenatally at the initial booking. Even using this relatively crude measure of continuity of care, mothers were more satisfied with the examination if the same midwife was present at antenatal booking and examined her newborn, rather than a different midwife or SHO.

Contacts during routine child health surveillance provide an important opportunity to provide health education and address parental concerns. ${ }^{132}$ The provision of adequate information has been found to be an important contributor to consumer satisfaction in various fields of health care..$^{21} 3011$ Providing healthcare advice-on feeding, skin care, infant sleeping, and stool and nappy care-was highly valued by mothers at this time. This was independent of whether it was provided by a midwife or doctor. However, during the examination, midwives $(61 \%)$ took the opportunity to discuss healthcare issues twice as often as did the SHOs (33\%), as reported by the mothers. Physical and behavioural care issues are the major parental concerns for otherwise healthy newborns at this time, and the examination of the newborn can provide an important opportunity for education on physical and emotional care of the infant. ${ }^{32} 29$
Women who have experienced miscarriages have been reported to be more anxious, both generally and specifically, about the possibility of something being wrong with the baby. ${ }^{33-35}$ Being treated sympathetically by the healthcare staff after miscarriage has been considered to be an important contributor to satisfaction with care. ${ }^{36}$ This study found that mothers who had suffered previous miscarriages were $58 \%$ less satisfied with the newborn examination, independent of whether carried out by an SHO or midwife. New mothers with a history of miscarriage may need special reassurance not currently provided during the newborn examination.

It is important to note that, according to the exclusion criteria agreed with the midwives and paediatricians at the trial hospital, only about half (53\%) of all newborns were eligible for midwife examination. Other and perhaps less stringent exclusion criteria may be considered in future. Furthermore, $11 \%$ of the newborns assigned to a midwife were assessed by an SHO, as the midwife was not available at the time. Midwives also took about five minutes longer to carry out the newborn examination than junior paediatricians, although time taken by itself did not positively influence maternal satisfaction. However, no differences in the rate of hospital referrals to a registrar, consultant, or outpatient department were found between midwife and SHO examinations (4\%). More than a quarter $(29 \%)$ of midwife examinations were carried out at home, allowing greater flexibility when discharging mothers and their newborn from hospital. These factors need to be taken into account if changes in the delivery of the newborn examination are considered.

\section{Methodological issues}

The major outcome measure was a seven item scale of satisfaction with the newborn examination which was specifically developed for this study. The internal reliability of the scale was high (0.94), exceeding that reported for patient satisfaction scales in investigations of maternity care ${ }^{30}$ or general practice. ${ }^{37}$ Principal component analysis and the low and non-significant correlations with other scales relating to satisfaction with labour and postnatal care $(r=-0.09)$ or maternal mood and bonding to her newborn $(r=0.15)$ indicate high construct and discriminant validity. That is, the major outcome measure assessed satisfaction specifically with the newborn examination independent of other care experiences or maternal wellbeing, and findings cannot be attributed to systematic bias. ${ }^{38}$ The scale developed and tested here may be highly suitable for assessing satisfaction with the newborn examination in clinical practice for audit purposes, or research.

The randomised control trial was analysed by intention to treat. However, to test whether other and examination specific factors explain maternal satisfaction ratings, analysis according to those who were actually treated by a midwife or SHO 
should be conducted. This analysis by actual treatment for the final maximum likelihood logistic regression model is thus presented in addition (table 4B). The results were virtually the same whether analysed by intention to treat or actual treatment and support the interpretation of the findings.

\section{Conclusions}

The results support the conclusion that, from the mother's perspective, the quality of midwife examination is at least as satisfactory as that of SHOs, when adequate training and paediatric support have been provided. Midwives more often provided information on healthcare issues that are important at this time for mothers, such as infant feeding, skin care, jaundice, sleeping, and stool and nappy care, thus enhancing the quality of care. Furthermore, midwives are able to provide continuity of care, which was found to increase maternal satisfaction with the newborn examination. It was apparent that more reassurance is required to increase satisfaction in new mothers who have had previous miscarriages.

On a cautious note, maternal satisfaction is important, but is only one of different indicators of quality of care. Full recommendations will be made when the study, including a current longitudinal evaluation on the safety, observed quality, and cost effectiveness of midwife versus SHO examination, as well as a national survey of current practice and qualitative analysis of stakeholders' views of the newborn examination and organisational implications, is completed.

\section{ACKNOWLEDGEMENTS}

The work was funded as part of the UK NHS Health Technology Assessment Programme (grant 94/40/05). Opinions and conclusions are those of the authors and not of the funders. Thanks are due to Anne Monument, Emmanuel Quist-Therson, Cathy Rogers, Linda Bloomfield, David Messer, and Powatti Ramchand, for comments and help with the study.

\section{Authors' affiliations}

D Wolke, Department of Psychology, University of Herffordshire, Hatfield, Herts, UK

S Dave, J Hayes, J Townsend, Centre for Research in Primary and Community Care, University of Hertfordshire

M Tomlin, Department of Midwifery and Child, University of Herffordshire

\section{REFERENCES}

1 Hall DMB. Health for all children. 3rd ed. Oxford: Oxford University Press, 1996.

2 Cartlidge PHT. Routine discharge examination of babies: is it necessary? Arch Dis Child 1992;67:1421-2.

3 Hall DMB. The role of the routine neonatal examination. BM 1999;318:619-20.

4 Glazener CMA, Ramsay CR, Campbell MK, et al. Neonatal examination and screening trial (NEST): a randomised, controlled, switchback trial of alternative policies for low risk infants. BM 1999;318:627-31.

5 Moss GD, Cartlidge PHT, Speidel BD, et al. Routine examination in the neonatal period. BM 1991;302:878-9.

6 Department of Health and Social Security. Screening for the detection of congenital dislocation of the hip. London: HMSO, 1996.

7 Platt WMP. Newborn screening examination (excluding congenital dislocation of the hip). Semin Neonatol 1998:3:61-6.

8 Ainsworth S, Wyllie JP, Wren C. Prevalence and clinical significance of cardiac murmurs in neonates. Arch Dis Child Fetal Neonatal Ed $1999 ; 80: F 43-5$
9 Dezateux C. Godward S. A national survey of screening for congenital dislocation of the hip. Arch Dis Child 1996;74:445-8

10 Department of Health. Changing childbirth. A report of the Expert Maternity Group. London: HMSO, 1993.

11 Rose SJ. Physical examination of the full-term newborn. British Journal of Midwifery 1994;2:209-13.

12 MacKeith N. Who should examine the 'normal' neonate. Nursing Times 1995;91:34-5.

13 University of Hertfordshire. ENB N96: the neuro-behavioural physiological assessment of the newborn. Department of Midwifery and Child, 2000.

14 Cleary PD, McNeil BJ. Patient satisfaction as an indicator of quality care. Inquiry 1988;25:25-36.

15 The NHS plan. A plan for investment. A plan for reform. London: HMSO 2000.

16 Delbanco TL. Quality of care through the patient's eyes. BM 1996:313:832-3.

17 Kerfoot KM, LeClair C. Nursing management considerations. Nursing Economics 1991;9:441-3.

18 Cottrell BH, Grubbs LM. Women's satisfaction with couplet care nursing compared to traditional postpartum care with rooming-in. Res Nurs Health 1994;17:401-9.

19 Wardle S. The Mid-Staffordshire survey. Getting consumers' views of maternity services. Professional Care of Mother \& Child 1994;4:170-4.

20 Salisbury C. Postal survey of patients' satisfaction with a general practice out of hours cooperative. BM 1997;314:1594.

21 Risser NL. Development of an instrument to measure patient satisfaction with nurses and nursing care in primary care settings. Nurs Res 1975:24:45-52.

22 Seguin L, Terrien R, Champagne F, et al. Components of women's satisfaction with maternity care. Birth 1989;16:109-13.

23 Spitzer RB. Meeting consumer expectations. Nurs Adm Q 1988;12:31-9.

24 Kenny P, King MT, Cameron S, Shiell A. Satisfaction with postnata care: the choice of home or hospital. Midwifery 1993;9:146-53.

25 Young D. First class delivery: the importance of asking women what they think about their maternity care. Birth 1998;25:71-2.

26 Williams SJ, Calnan M. Key determinants of consumer satisfaction with general practice. Fam Pract 1991;8:237-42.

27 Morgan M, Fenwick N, McKenzie C, et al. Quality of midwifery led care: assessing the effects of different models of continuity for women's satisfaction. Qual Health Care 1998:7:77-82.

28 Wolke D. Parents' perceptions as guides for conducting NBAS clinical sessions. In: Brazelton TB, Nugent JK, eds. Neonatal behavioral assessment scale. 3rd ed. Cambridge: Cambridge University Press, 1995: 117-25.

29 Walker $\mathbf{D}$. Role of the routine neonatal examination. BM 1999;318: 1766

30 Bramadat IJ, Driedger RN. Satisfaction with childbirth: theories and methods of measurement. Birth 1991;20:22-9.

31 Proctor S. What determines quality in maternity care? Comprising the perceptions of childbearing women and midwives. Birth 1998;25:85-93

32 Wolke D, Söhne B, Riegel K, et al. An epidemiologic longitudinal study of sleeping problems and feeding experience of preterm and term children in southern Finland: comparison with a southern German population sample. J Pediatr 1998;133:224-31

33 Statham H, Green JM. The effects of miscarriage and other 'unsuccessful' pregnancies on feelings early in a subsequent pregnancy. J Reprod Infant Psychol 1994; 12:45-54.

34 Slade P. Predicting the psychological impact of miscarriage. J Reprod Infant Psychol 1994;12:5-16.

35 Klock SC, Chang G, Hiley AH, et al. Psychological distress among women with recurrent spontaneous abortion. Psychomatics 1997;38:503-7.

36 Moohan J, Ashe RG, Cecil R. The management of miscarriage: results from a survey at one hospital. J Reprod Infant Psychol 1994;12:17-19.

37 McKinley RK, Manku-Scott T, Hastings AM, et al. Reliability and validity of a new measure of patient satisfaction with out of hours primary medical care in the United Kingdom: development of a patient questionnaire. BM 1997;314:193-8.

38 Streiner DL, Norman GR. Health measurement scales. A practical guide to their development and use. Oxford: Oxford Medical Publications, 1989. 